\title{
AN OBSERVED LACK OF SUBSTRUCTURE IN STARLESS CORES
}

\author{
Scott Schnee ${ }^{1}$, Melissa Enoch ${ }^{2}$, Doug Johnstone ${ }^{1,3}$, Thomas Culverhouse ${ }^{4,5}$, Erik Leitch $^{4,5}$, Daniel P. Marrone ${ }^{4,5}$, \\ AND ANNEILA SARGENT 6 \\ ${ }^{1}$ National Research Council Canada, Herzberg Institute of Astrophysics, 5071 West Saanich Road Victoria, BC V9E 2E7, Canada; scott.schnee@ nrc-cnrc.gc.ca \\ ${ }^{2}$ Department of Astronomy, University of California, Berkeley, CA 94720, USA \\ ${ }^{3}$ Department of Physics \& Astronomy, University of Victoria, Victoria, BC, V8P 1A1, Canada \\ ${ }^{4}$ Kavli Institute for Cosmological Physics, Department of Astronomy and Astrophysics, University of Chicago, Chicago, IL 60637, USA \\ ${ }^{5}$ Department of Astronomy and Astrophysics, University of Chicago, Chicago, IL 60637, USA \\ ${ }^{6}$ Department of Astronomy, California Institute of Technology, MC 105-24 Pasadena, CA 91125, USA \\ Received 2010 March 12; accepted 2010 May 26; published 2010 June 29
}

\begin{abstract}
In this paper, we present the results of a high resolution (5") Combined Array for Research in Millimeter-wave Astronomy and Sunyaev-Zeldovich Array survey of the $3 \mathrm{~mm}$ continuum emission from 11 of the brightest (at $1.1 \mathrm{~mm}$ ) starless cores in the Perseus molecular cloud. We detect 2 of the 11 cores, both of which are composed of single structures, and the median $3 \sigma$ upper limit for the non-detections is $0.2 M_{\odot}$ in a $\sim 5^{\prime \prime}$ beam. These results are consistent with, and as stringent as, the low detection rate of compact $3 \mathrm{~mm}$ continuum emission in dense cores in Perseus reported by Olmi et al. From the non-detection of multiple components in any of the 11 cores, we conclude that starless core mass functions derived from bolometer maps at resolutions range $10^{\prime \prime}-30^{\prime \prime}$ (e.g., with MAMBO, SCUBA, or Bolocam) are unlikely to be significantly biased by the blending of lower mass cores with small separations. These observations provide additional evidence that the majority of starless cores in Perseus have inner density profiles shallower than $r^{-2}$.
\end{abstract}

Key words: dust, extinction - stars: formation

Online-only material: color figures

\section{INTRODUCTION}

Surveys of starless cores in nearby molecular clouds have shown that the starless core mass function (CMF) looks very similar to the stellar initial mass function (IMF) but translated toward higher masses by a factor of a few (e.g., Motte et al. 1998; Nutter \& Ward-Thompson 2007; Enoch et al. 2008; Simpson et al. 2008). If each starless core collapses into a single star, then the apparent shift between the IMF and the CMF implies that the formation process is inefficient and most of the mass in each core never makes it into any star. Models have shown that $25 \%-70 \%$ of the mass in a protostellar core will be converted into a star with the remaining material being ejected by outflows (Matzner \& McKee 2000). A second reason for the shift between the CMF and the IMF is likely to be fragmentation, in which a single starless core will eventually form multiple stars. Since many stars are found in binary or higher-order systems (e.g., Duquennoy \& Mayor 1991), to some extent the fragmentation of a starless core into multiple bound objects is expected. However, lower mass stars are less frequently found in gravitationally bound systems (Lada 2006).

Although the shape of the IMF can be reproduced from the CMF with a variety of assumptions about the multiplicity, fragmentation, and efficiency of the process (Swift \& Williams 2008; Hatchell \& Fuller 2008), recent work by Goodwin et al. (2008) has shown that models in which every starless core eventually forms multiple stars reproduce the stellar IMF more successfully than models in which low-mass cores are more likely to form a single star than a binary. That each core fragments into only two or three components, and not many more, is suggested by the relatively small number of close binaries and high velocity single stars that would result from the dynamical ejection of protostars from higher order systems (Goodwin \& Kroupa 2005). Therefore, high angular resolution observations of starless cores might be expected to show that what appears to be a single object at low resolution is in fact composed of multiple fragments, depending on whether or not the fragmentation takes place before an embedded protostar forms and on what physical scale fragmentation occurs.

The binary fraction of young stellar objects and protostars has been studied in detail, and it has been found that these objects are commonly found in multiple systems. For example, nearinfrared observations of Class-I and flat-spectrum objects in nearby molecular clouds show that in the range 300-2000 AU the multiplicity is $\sim 18 \%$, with a median separation between companions of $\sim 900 \mathrm{AU}$ (Haisch et al. 2004). Sub-arcsecond Berkeley-Illinois-Maryland Array (BIMA) observations of the $3 \mathrm{~mm}$ continuum emission from 20 protostars (Class 0 and Class I) found that all of the embedded objects are in small groupings, with separations ranging from $<100 \mathrm{AU}$ to $>10000$ AU (Looney et al. 2000). Recent simulations of fragmentation in magnetically supported dense cores typically start with small cores ( $2000 \mathrm{AU}$ or $24^{\prime \prime}$ at the distance of Perseus) that fragment into multiple protostellar systems with separations of the order of 10-1000 AU (e.g., Boss 2002, 2007; Price $\&$ Bate 2007).

Observations of starless cores have not often found evidence for fragmentation on the scale of $5^{\prime \prime}-10^{\prime \prime}$. In a survey of dense cores in the Perseus molecular cloud with the Owens Valley Radio Observatory (OVRO) interferometer, Olmi et al. (2005) found that five out of six cores had no compact continuum emission and in the sixth core a single source was detected (at $\sim 5^{\prime \prime}$ resolution or $1200 \mathrm{AU}$ in Perseus). Similarly, Harvey et al. (2003) mapped the $1.3 \mathrm{~mm}$ continuum emission from the starless core L694-2 with the IRAM Plateau de Bure Interferometer (PdBI) and BIMA and found that the PdBI observations resolved out the core entirely while the shorter baselines of the BIMA data can be adequately modeled by a single source, implying 
Table 1

Core Properties from Bolocam $1 \mathrm{~mm}$ Data

\begin{tabular}{|c|c|c|c|c|c|c|c|}
\hline Name & $\begin{array}{c}\text { R.A. } \\
\text { (J2000) }\end{array}$ & $\begin{array}{c}\text { Decl. } \\
\text { (J2000) }\end{array}$ & $\begin{array}{c}\text { Peak Flux } \\
\left(\mathrm{mJy} \mathrm{beam}^{-1}\right)\end{array}$ & $\begin{array}{c}\text { Total Flux } \\
(\mathrm{Jy})\end{array}$ & $\begin{array}{c}\text { Total Mass } \\
\left(M_{\odot}\right)\end{array}$ & $\begin{array}{c}\text { FWHM }^{\mathrm{a}} \\
\left({ }^{\prime \prime}\right)\end{array}$ & $\begin{array}{c}\text { Density }^{\mathrm{b}} \\
\left(10^{5} \mathrm{~cm}^{-3}\right)\end{array}$ \\
\hline Perbo11 & $03: 25: 46.0$ & $+30: 44: 10$ & 241 & 0.90 & 2.18 & 72.5 & 2.2 \\
\hline Perbo13 & $03: 25: 48.8$ & $+30: 42: 24$ & 407 & 0.47 & 1.14 & 38.2 & 3.9 \\
\hline Perbo14 & $03: 25: 50.6$ & $+30: 42: 01$ & 342 & 0.41 & 1.00 & 38.7 & 3.5 \\
\hline Perbo44 & $03: 29: 04.5$ & $+31: 18: 42$ & 274 & 0.72 & 1.73 & 47.0 & 2.7 \\
\hline Perbo45 & 03:29:07.7 & $+31: 17: 17$ & 455 & 1.35 & 3.25 & 52.9 & 4.8 \\
\hline Perbo50 & $03: 29: 14.5$ & $+31: 20: 30$ & 313 & 1.31 & 3.15 & 68.0 & 3.2 \\
\hline Perbo51 & $03: 29: 17.0$ & $+31: 12: 26$ & 423 & 0.62 & 1.49 & 34.7 & 3.6 \\
\hline Perbo58 & $03: 29: 25.7$ & $+31: 28: 16$ & 273 & 0.33 & 0.78 & 25.9 & 1.8 \\
\hline Perbo74 & $03: 33: 01.9$ & $+31: 04: 32$ & 255 & 0.38 & 0.91 & 44.5 & 2.6 \\
\hline Perbo105 & $03: 43: 57.8$ & $+32: 04: 06$ & 283 & 0.61 & 1.47 & 46.6 & 2.8 \\
\hline Perbo107 & 03:44:02.1 & $+32: 02: 34$ & 388 & 0.48 & 1.17 & 46.0 & 4.1 \\
\hline
\end{tabular}

Notes.

a Derived from an elliptical Gaussian fit, deconvolved by the $31^{\prime \prime}$ beam.

${ }^{\mathrm{b}}$ Mean density calculated in a fixed linear aperture of diameter $10^{4} \mathrm{AU}$.

that no fragmentation has taken place. Other interferometric observations of starless cores, such as S68NW, L1544, and L694-2, show only single peaks in their integrated spectral line emission (Williams \& Myers 1999; Williams et al. 1999, 2006). However, BIMA mapping of the $\mathrm{N}_{2} \mathrm{H}^{+}$emission from the starless core L183 shows that this core is composed of three components with a total mass of a few tenths of a solar mass that are distinct both spatially and kinematically (Kirk et al. 2009). Kirk et al. (2009) interpret this as evidence that L183 is fragmenting as it collapses and predict that this core will eventually form multiple protostars.

Here, we present the results of a search for substructure in a sample of 11 starless cores in the Perseus molecular cloud. We use Combined Array for Research in Millimeter-wave Astronomy (CARMA) and Sunyaev-Zel'dovich Array (SZA) interferometric observations to study the $3 \mathrm{~mm}$ continuum emission at $\sim 5^{\prime \prime}$ resolution with sensitivity up to scales of $30^{\prime \prime}-80^{\prime \prime}$. The source selection and observations are discussed in Section 2, followed by an analysis (Section 3), a discussion (Section 4), and summary (Section 5).

\section{OBSERVATIONS}

To search for substructure within starless cores, we mapped the $3 \mathrm{~mm}$ continuum emission with $\sim 5^{\prime \prime}$ resolution toward a sample of 11 cores drawn from the $1.1 \mathrm{~mm}$ Bolocam survey of Enoch et al. (2008). Here, we describe the sample of cores and their observations.

\subsection{Source Selection}

We drew our sample of starless cores from the $1.1 \mathrm{~mm}$ Bolocam survey of Enoch et al. (2006, 2008), which has $31^{\prime \prime}$ resolution. Enoch et al. (2008) use Spitzer near- to midinfrared data to determine the starless/protostellar status of each millimeter core. We chose starless cores in the Perseus molecular cloud on the basis of having high peak fluxes $\left(>200 \mathrm{mJy}\right.$ beam $\left.^{-1}\right)$. There are 15 starless cores in the Bolocam survey of Perseus with peak fluxes $>200 \mathrm{mJy} \mathrm{beam}^{-1}$, of which we were allocated enough time to observe only 11 , which were chosen arbitrarily. The selected cores have a median mass of $1.47 M_{\odot}$ and a median density of $3.2 \times 10^{5} \mathrm{~cm}^{-3}$, as derived from the Bolocam data (Enoch et al. 2008). The $1.1 \mathrm{~mm}$-derived properties of the starless cores in this sample are presented in Table 1.
Protostars in Enoch et al. (2008) are identified by the shape of their near-infrared to far-infrared spectral energy distributions, minimum flux at $24 \mu \mathrm{m}$, and presence of $70 \mu \mathrm{m}$ point sources not classified as galaxy candidates. In Enoch et al. (2008), if a protostar is found within one FWHM of the center of the $1.1 \mathrm{~mm}$ Bolocam core, then it is classified as protostellar, otherwise the Bolocam core is classified as starless. Searches for protostars in the Perseus molecular cloud have also been performed by Jørgensen et al. (2007), Hatchell et al. (2007), and Sadavoy et al. (2010). In Jørgensen et al. (2007), SCUBA cores are classified as protostellar based on the colors and magnitudes of nearby mid-infrared sources, the distance between the midinfrared source and SCUBA core, and the concentration of the $850 \mu \mathrm{m}$ emission. Hatchell et al. (2007) identify protostellar cores by the presence of ${ }^{12} \mathrm{CO}$ outflows and the presence of nearinfrared (Two Micron All Sky Survey) or mid-infrared (Spitzer) sources within an $850 \mu \mathrm{m}$ SCUBA core. Sadavoy et al. (2010) find protostars based on mid-infrared and far-infrared (Spitzer) magnitudes and colors cross-referenced with a list of $850 \mu \mathrm{m}$ SCUBA cores. A detailed comparison between these methods of classifying a core as either starless or protostellar is given in Sadavoy et al. (2010); here, we note that the classifications according to the various criteria are broadly consistent.

All of the cores in our sample are classified as starless by Enoch et al. (2008) and none of these cores are identified as protostellar by Jørgensen et al. (2007), but Perbo45 and Perbo50 are identified as Class 0 protostars by Hatchell et al. (2007) and Perbo44 and Perbo107 are identified as protostars by Sadavoy et al. (2010). More recent deep $70 \mu \mathrm{m}$ observations of one starless core in our sample, Perbo58, indicate that it may contain a very weak internal luminosity source (M. L. Enoch et al. 2010, in preparation). Thus, Perbo58 may not be truly prestellar, although even if a central protostar has formed, it is likely very young and not far evolved from the prestellar phase. We keep Perbo44, Perbo45, Perbo50, Perbo58, and Perbo107 in the starless sample based on the original selection criteria and note that out of the four surveys to identify starless cores and/or protostars in the Perseus molecular cloud none of our targets are classified as protostellar in more than one paper.

\subsection{CARMA and SZA}

Continuum observations in the $3 \mathrm{~mm}$ window were obtained with CARMA, a 15 element interferometer consisting of nine 
Table 2

$3 \mathrm{~mm}$ Observations

\begin{tabular}{|c|c|c|c|c|c|c|}
\hline Name & CARMA D & CARMA E & SZA & $\begin{array}{c}\text { Beam Size }{ }^{a} \\
\left({ }^{\prime \prime}\right)\end{array}$ & $\begin{array}{l}\mathrm{LAS}^{\mathrm{b}} \\
\left({ }^{\prime \prime}\right)\end{array}$ & $\begin{array}{c}\text { Noise } \\
\left(\mathrm{mJy}^{2} \text { beam }^{-1}\right)\end{array}$ \\
\hline Perbo11 & $\mathrm{Y}$ & $\mathrm{N}$ & $\mathrm{N}$ & 4.5 & 30 & 0.5 \\
\hline Perbo13 & $\mathrm{Y}$ & $\mathrm{Y}$ & $\mathrm{N}$ & 6.6 & 40 & 1.3 \\
\hline Perbo14 & $\mathrm{Y}$ & $\mathrm{Y}$ & $\mathrm{N}$ & 6.2 & 40 & 0.9 \\
\hline Perbo44 & $\mathrm{Y}$ & $\mathrm{N}$ & $\mathrm{N}$ & 4.2 & 30 & 0.6 \\
\hline Perbo45 & $\mathrm{Y}$ & $\mathrm{Y}$ & $\mathrm{N}$ & 6.4 & 40 & 0.4 \\
\hline Perbo50 & $\mathrm{Y}$ & $\mathrm{N}$ & $\mathrm{N}$ & 4.2 & 30 & 0.7 \\
\hline Perbo5 $1^{\mathrm{c}}$ & Y & $\mathrm{Y}$ & $\mathrm{N}$ & 5.4 & 40 & 2.8 \\
\hline Perbo58 & $\mathrm{Y}$ & $\mathrm{Y}$ & $\mathrm{Y}$ & 5.6 & 80 & 0.3 \\
\hline Perbo74 & Y & $\mathrm{N}$ & $\mathrm{N}$ & 4.6 & 30 & 0.3 \\
\hline Perbo105 & Y & $\mathrm{N}$ & $\mathrm{N}$ & 4.3 & 30 & 0.9 \\
\hline Perbo107 & $\mathrm{Y}$ & $\mathrm{Y}$ & $\mathrm{N}$ & 6.2 & 40 & 1.1 \\
\hline
\end{tabular}

Notes.

${ }^{a}$ Geometric mean of the major and minor axes.

b Largest angular scale to which the map is sensitive.

${ }^{c}$ The noise in the map of Perbo51 is larger than the other maps due to the bright source NGC 1333 IRAS4 located at the edge of the CARMA map.

6.1 $\mathrm{m}$ antennas and six $10.4 \mathrm{~m}$ antennas. The CARMA correlator records signals in three separate bands, each with an upper and lower sideband. We configured all three bands for maximum bandwidth (468 MHz with 15 channels per band) to observe continuum emission, providing a total continuum bandwidth of $2.8 \mathrm{GHz}$. The observations were centered around $102 \mathrm{GHz}$ and range from $100 \mathrm{GHz}$ to $104 \mathrm{GHz}$. The half-power beam width of the $10.4 \mathrm{~m}$ antennas is $66^{\prime \prime}$ at the observed frequencies. Single pointings toward all 11 starless cores were taken in the CARMA D-array configuration, with projected baselines that range from $11 \mathrm{~m}$ to $150 \mathrm{~m}$. Follow-up observations of 6 of the 11 cores were made in the CARMA E-array configuration with projected baselines that range from $8 \mathrm{~m}$ to $66 \mathrm{~m}$, also with single pointings. Two cores (Perbo45 and Perbo58) were then re-observed in D- and E-array configurations with 7-point mosaics to improve the signal-to-noise ratio in the maps and enlarge the area of uniform gain to 66". A summary of the CARMA configurations, the synthesized beam size, the largest angular scale to which the observations are sensitive, and the noise in the maps are presented in Table 2.

The SZA is an 8-element interferometer equipped with $3 \mathrm{~mm}$ band $(80-115 \mathrm{GHz})$ receivers. The instantaneous field of view of the SZA is given by the primary beam of the $3.5 \mathrm{~m}$ antennas, approximately 3.3 (FWHM) at $95 \mathrm{GHz}$. Six of the SZA antennas are arranged in a close-packed configuration with baselines ranging from 4.5 to $11.5 \mathrm{~m}$, and two outer telescopes yield baselines of up to $65 \mathrm{~m}$, resulting in a synthesized beam size of $38^{\prime \prime}$. Sixteen $500 \mathrm{MHz}$-wide analog bands provide $8 \mathrm{GHz}$ bandwidth, though for the observations in this study only $7 \mathrm{GHz}$ bandwidth (from 92 to $98 \mathrm{GHz}$ ) was available. A more detailed description of the SZA is given in Muchovej et al. (2007).

The observing sequence for the CARMA observations was to integrate on a phase calibrator $(3 \mathrm{C} 84,0237+288$, or $0336+323)$ for 4-5 minutes and science targets for 14-28 minutes. In each set of observations, 3C84 was observed for passband calibration, and observations of a planet (Mars or Uranus) or 3C84 were used for absolute flux calibration. Although 3C84 has a variable brightness, it is monitored on a weekly basis at CARMA. Based on the repeatability of the quasar fluxes, the estimated random uncertainty in the measured source fluxes is $\sigma \sim 5 \%$. Radio pointing was done at the beginning of each track and pointing constants were updated at least every two hours thereafter, using

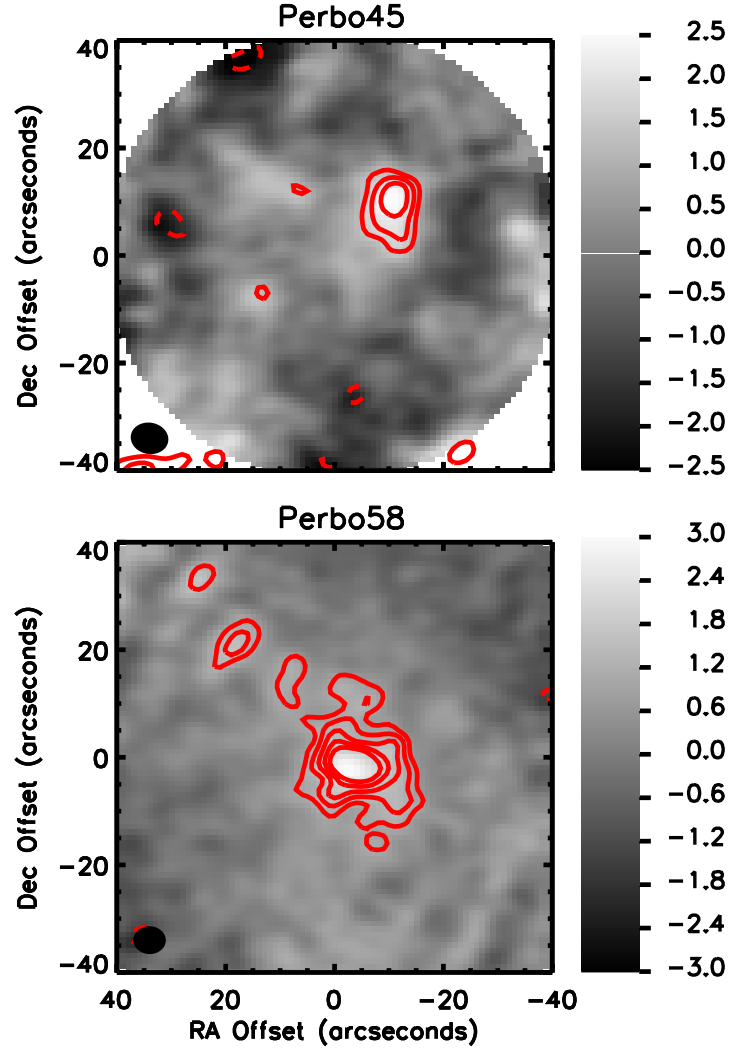

Figure 1. $3 \mathrm{~mm}$ flux maps (gray scale and contours) of Perbo45 (top) and Perbo58 (bottom), from data taken with CARMA and SZA. Contours start at $3 \sigma$ and increase by $1 \sigma$, negative peaks are shown with dashed contours. The beam size is shown in the bottom left corner of each map. The units of the maps are mJy beam ${ }^{-1}$.

(A color version of this figure is available in the online journal.)

either radio or optical pointing routines. Calibration and imaging were done using the MIRIAD data reduction package (Sault et al. 1995).

The observing sequence for the SZA observations was to integrate on the phase calibrator 3C84 for five minutes and the starless core Perbo58 for ten minutes. The observations of 3C84 were also used for bandpass and absolute flux calibration. Because the SZA data are at a somewhat lower frequency than the CARMA data (95 GHz vs. $102 \mathrm{GHz}$ ), we use the flux of 3C84 derived at the higher frequency to set the absolute flux calibration of the SZA data before combining the CARMA and SZA maps. Calibration and imaging were done using the MIRIAD data reduction package (Sault et al. 1995).

The $3 \mathrm{~mm}$ continuum maps of Perbo45 and Perbo58 are presented in Figure 1. The other starless cores were not detected.

\subsection{Bolometer Maps}

In addition to the $31^{\prime \prime}$ Bolocam map at $1100 \mu \mathrm{m}$ (Enoch et al. 2006), the Perseus molecular cloud has also been mapped with SCUBA at 450 and $850 \mu \mathrm{m}$ at $9^{\prime \prime}$ and $14^{\prime \prime}$ resolution, respectively (Hatchell et al. 2005; Kirk et al. 2006). We downloaded the submillimeter maps from the SCUBA Legacy Catalogues (Di Francesco et al. 2008), which includes all of the data taken with SCUBA and uses the most current calibration and reduction methods. The absolute calibration of the bolometer maps is good to $15 \%, 20 \%$, and $50 \%$ at 1100,850 , and $450 \mu \mathrm{m}$, respectively. The 450, 850, and $1100 \mu \mathrm{m}$ bolometer maps of Perbo45 and Perbo58 are overlaid on the $3 \mathrm{~mm}$ interferometric maps in Figure 2. 

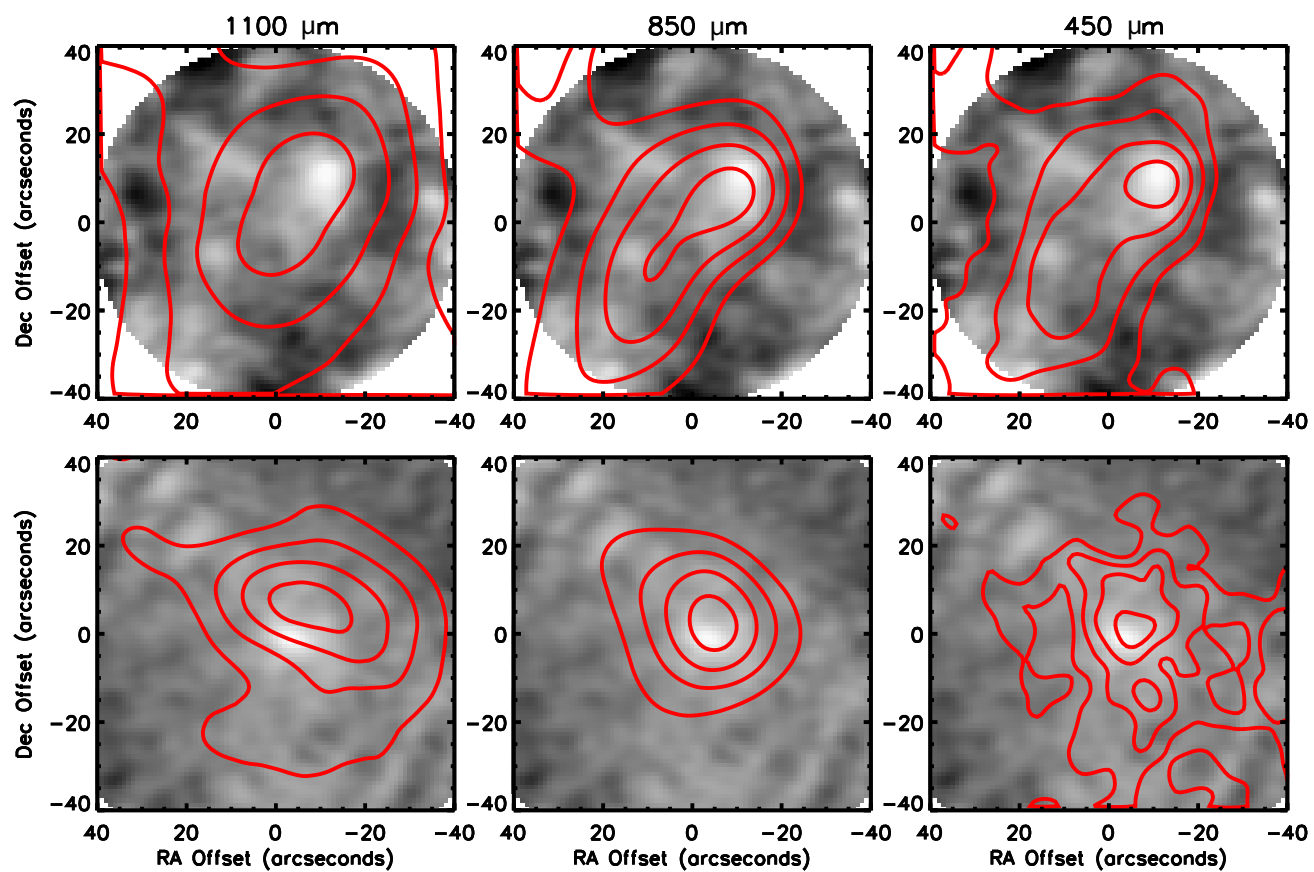

Figure 2. 3 mm flux maps (gray scale) of Perbo45 (top) and Perbo58 (bottom), with the same scale as in Figure 1. Contours show the emission from maps made by Bolocam $1.1 \mathrm{~mm}$ (left), SCUBA $850 \mu \mathrm{m}$ (middle), and SCUBA $450 \mu \mathrm{m}$ (right), with contour levels at 30\%, 50\%, 70\%, and 90\% of the peak in each map.

(A color version of this figure is available in the online journal.)

\section{ANALYSIS}

We detected, at the $\geqslant 5 \sigma$ level, continuum emission from 2 out of 11 starless cores in our survey. Here, we present an analysis of the properties of the detected cores and the implications of this detection rate.

\subsection{Core Properties}

Both of the detected cores in this survey (Perbo45 and Perbo58) are seen to contain one main component (see Figure 1). A few lower significance $(3 \sigma-4 \sigma)$ peaks are present in the $3 \mathrm{~mm}$ map of Perbo58 along an extended ridge seen in the bolometer maps. Note that the map of Perbo58 includes data taken with the SZA, so it is sensitive to angular scales smaller than $\sim 80^{\prime \prime}$. Given that the FWHM sizes of Perbo58 as measured by Bolocam (26") are smaller than this scale we believe that we have not resolved out any of the $3 \mathrm{~mm}$ emission from Perbo58, but given that the map of Perbo45 only includes CARMA D+E array data and not SZA data we are likely resolving out some of the $3 \mathrm{~mm}$ emission from Perbo45.

\subsubsection{Mass}

We calculate the mass of the detected cores from the total $3 \mathrm{~mm}$ flux and upper limits to the non-detections using the equation:

$$
M=\frac{d^{2} S_{3 \mathrm{~mm}}}{B_{v}\left(T_{D}\right) \kappa_{3 \mathrm{~mm}}}
$$

where $d$ is the distance to Perseus, $S_{3 \mathrm{~mm}}$ is the total flux from each detection or three times the noise for the nondetections, $B_{v}$ is the Planck function at dust temperature $T_{D}$, and $\kappa_{3 \mathrm{~mm}}=0.00169 \mathrm{~cm}^{2} \mathrm{~g}^{-1}$ is the dust opacity, extrapolated from the opacity at $1300 \mu \mathrm{m}$ given in Ossenkopf \& Henning (1994; Table 1, Column (5)) for dust grains with thin ice mantles, assuming a gas-to-dust ratio of 100 and an emissivity given by $\beta=2$. This value of the emissivity spectral index is consistent with the measured $\beta$ in TMC-1C (Schnee et al. 2010) and L1498 (Shirley et al. 2005). For comparison with the core properties derived in Enoch et al. (2008), we assume that the distance to Perseus is $250 \mathrm{pc}$ and that the dust temperature is $10 \mathrm{~K}$ for all of the cores in this sample, which is a typical gas temperature of starless cores in Perseus (Schnee et al. 2009). The derived masses and upper limits are presented in Table 3.

The mass of Perbo45 derived from our $3 \mathrm{~mm}$ data is about a factor of 4 lower than the mass derived from the $1.1 \mathrm{~mm}$ Bolocam data. The mass of Perbo58 derived from our $3 \mathrm{~mm}$ observations is a factor of 3 higher than the mass derived from the $1.1 \mathrm{~mm}$ Bolocam data. Four possibilities for explaining these discrepancies are (1) uncertainties in the dust properties, (2) variations in the temperature within the cores, (3) errors in the measurement of the $1.1 \mathrm{~mm}$ and/or $3 \mathrm{~mm}$ fluxes, and (4) that in the case of Perbo45, we are likely to have resolved out some of the $3 \mathrm{~mm}$ continuum emission. Note that the deconvolved size of Perbo45 in the Bolocam surveys is roughly 53", so the amount of flux resolved out by our CARMA observations is likely to be substantial.

The masses of Perbo58 derived from the $1.1 \mathrm{~mm}$ and $3 \mathrm{~mm}$ data could be brought into agreement if the emissivity spectral index were given by $\beta \simeq 0.5-1$ instead of $\beta=2$, as we assumed. Although the two best measurements of the emissivity spectral index in starless cores suggest that $\beta \geqslant 2$ is a much better fit than $\beta \leqslant 1$ (Shirley et al. 2005; Schnee et al. 2010), it is possible that grain growth leads to a shallower emissivity spectral index at the high densities likely to be found at the centers of prestellar cores. For instance, the emissivity spectral index in a sample of nearby protostars has been measured to be in the range 0.25-1.5 (Arce \& Sargent 2006), and values in this range might be plausible for starless cores given the recent evidence for grain growth in starless cores (Steinacker et al. 2010). Because the masses in Table 3 are derived from the $1.3 \mathrm{~mm}$ opacity (Ossenkopf \& Henning 1994) extrapolated out to $3 \mathrm{~mm}$, an incorrect assumption about the emissivity spectral 
Table 3

Gaussian Fits to $3 \mathrm{~mm}$ Observations

\begin{tabular}{|c|c|c|c|c|c|c|c|c|}
\hline Name & $\begin{array}{c}\text { R.A. Offset }{ }^{\mathrm{a}} \\
\left({ }^{\prime \prime}\right)\end{array}$ & $\begin{array}{c}\text { Decl. Offset } \\
\left({ }^{b}\right)\end{array}$ & $\begin{array}{c}\text { Peak Flux }{ }^{b} \\
\left(\mathrm{mJy} \mathrm{beam}^{-1}\right)\end{array}$ & $\begin{array}{c}\text { Total Flux } \\
(\mathrm{mJy})\end{array}$ & $\begin{array}{c}\text { Axes }^{\mathrm{c}} \\
\left({ }^{\prime \prime}\right)\end{array}$ & $\begin{array}{c}\theta_{P A}{ }^{\mathrm{c}} \\
\text { (degrees) }\end{array}$ & $\begin{array}{l}\text { Mass }^{\mathrm{d}} \\
\left(M_{\odot}\right)\end{array}$ & $\begin{array}{l}\text { Density } \\
\left(\mathrm{cm}^{-3}\right)\end{array}$ \\
\hline Perbo11 & & & & & & & $<0.11$ & \\
\hline Perbo13 & & & & & & & $<0.29$ & \\
\hline Perbo14 & & & & & & & $<0.20$ & \\
\hline Perbo44 & & & & & & & $<0.14$ & \\
\hline Perbo45 & $-10.3 \pm 0.5$ & $8.8 \pm 0.7$ & $2.4 \pm 0.3$ & $11 \pm 0.5$ & $14 \times 9$ & -14 & 0.8 & $1.1 \times 10^{7}$ \\
\hline Perbo50 & & & & & & & $<0.16$ & \\
\hline Perbo51 & & & & & & & $<0.62$ & \\
\hline Perbo58 & $-4.3 \pm 0.8$ & $-1.1 \pm 0.9$ & $2.0 \pm 0.3$ & $33 \pm 1$ & $26 \times 18$ & 35 & 2.4 & $4.5 \times 10^{6}$ \\
\hline Perbo74 & & & & & & & $<0.07$ & \\
\hline Perbo105 & & & & & & & $<0.20$ & \\
\hline Perbo107 & & & & & & & $<0.24$ & \\
\hline
\end{tabular}

Notes.

${ }^{a}$ Offset measured from pointing center, given in Table 1.

$\mathrm{b}$ Errors derived from the random noise in the maps and do not include absolute flux calibration uncertainties.

${ }^{c}$ Deconvolved major and minor axes and position angle.

${ }^{\mathrm{d}}$ For non-detections, the $3 \sigma$ upper limit to a point source mass is given.

e Note that only CARMA D- and E-array observations have been taken for this source, so we are likely to have resolved out a larger fraction of the emission than for Perbo58.

index would lead to masses off by a factor of a few. If the emissivity spectral index in starless cores is closer to $\beta=1$ than $\beta=2$, then the expected fluxes at $3 \mathrm{~mm}$ as extrapolated from the $1.1 \mathrm{~mm}$ Bolocam observations would be a factor of $\sim 3$ larger than we have assumed. In this case, the non-detection of $3 \mathrm{~mm}$ continuum emission toward nine of the eleven cores in our sample becomes an even more significant result (see Section 3.2).

Alternatively, if Perbo58 were significantly warmer at the center than its average temperature, the higher resolution $3 \mathrm{~mm}$ observations would be more biased toward high masses and densities than the low resolution $1.1 \mathrm{~mm}$ data. It is generally observed that starless cores are colder at their centers due to self-shielding (e.g., Crapsi et al. 2007; Schnee et al. 2007). Heating due to contraction can result in temperatures a few degrees higher in the center of a starless core (Keto \& Caselli 2010), but this on its own cannot account for the large difference in masses derived from the $1.1 \mathrm{~mm}$ and $3 \mathrm{~mm}$ continuum data. If there were a weak internal luminosity source in Perbo58 (as discussed in Section 2.1), then one would expect some central heating that could bring the $1.1 \mathrm{~mm}$ - and $3 \mathrm{~mm}$-derived masses into agreement.

A third possible cause for the disagreement between the $1.1 \mathrm{~mm}$ - and $3 \mathrm{~mm}$-derived masses is the uncertain absolute calibration of the emission maps. The two cores detected by CARMA/SZA are both in the dense and bright cluster NGC1333, which results in artifacts in the bolometer maps. However, detailed testing of the accuracy of the recovery of $1.1 \mathrm{~mm}$ flux from Bolocam has shown that the quoted fluxes are accurate to within 15\% (Enoch et al. 2006). The absolute calibration of the $3 \mathrm{~mm}$ continuum maps is accurate to within $20 \%$. Therefore, uncertainties in the absolute calibration of the emission maps alone cannot account for the factor of a few difference between the $1.1 \mathrm{~mm}$ - and $3 \mathrm{~mm}$-derived masses.

Due to uncertainties in the dust emissivity and temperature profiles of Perbo45 and Perbo58, we will defer a more detailed analysis of their masses until more data are available and only note that the $3 \mathrm{~mm}$ fluxes of Perbo45 and Perbo58 are not what we expected based on their $1.1 \mathrm{~mm}$ fluxes as mapped by Bolocam.

\subsubsection{Density}

We calculate the density of the detected starless cores from the derived mass and effective radius using the equation:

$$
n=\frac{3 M}{4 \pi r_{\mathrm{eff}}^{3} \mu m_{H}},
$$

where $r_{\text {eff }}$ is the effective radius, $\mu=2.33$ is the mean molecular weight per particle, and $m_{H}$ is the mass of hydrogen. The effective radius is the geometric mean of the deconvolved semimajor and semi-minor axes, derived from a Gaussian fit to the flux distribution. The density for each detected core is presented in Table 3.

The densities of Perbo45 and Perbo58 derived from the $3 \mathrm{~mm}$ continuum data in this survey are larger than the values derived from the $1.1 \mathrm{~mm}$ Bolocam data. The densities derived from the $3 \mathrm{~mm}$ data (see Table 3) are roughly a few $\times 10^{6}-10^{7} \mathrm{~cm}^{-3}$, while the mean density in a $10^{4} \mathrm{AU}$-diameter aperture derived from the $1.1 \mathrm{~mm}$ data is of the order of a few $\times 10^{5} \mathrm{~cm}^{-3}$. Since the $3 \mathrm{~mm}$ observations are higher resolution than the $1.1 \mathrm{~mm}$ observations, it is not surprising that the $3 \mathrm{~mm}$-derived densities are larger. Though high, densities in the $10^{6}-10^{7} \mathrm{~cm}^{-3}$ range have been reported in L1544 (Crapsi et al. 2007) and in several starless cores in Orion (Nutter \& Ward-Thompson 2007). The densities in Table 3 would be lower if the assumed temperature were higher, as might be expected if the detected cores harbor low-luminosity protostars.

A simple model of the density profile of a starless core can be given by:

$$
n(r)=\frac{n_{0}}{1+\left(\frac{r}{r_{0}}\right)^{\alpha}},
$$

where $n_{0}, r_{0}$, and $\alpha$ are constants. This formulation with $\alpha=2.5$ is a good approximation for the density profile of a Bonnor-Ebert sphere (Tafalla et al. 2004) and provides a qualitatively good fit to the observed density profiles of starless cores, which are fairly flat at small radii and are steeper at larger radii.

We investigate whether or not the density profiles of the cores in Perbo45 and Perbo58 are consistent with Equation (3). First, 

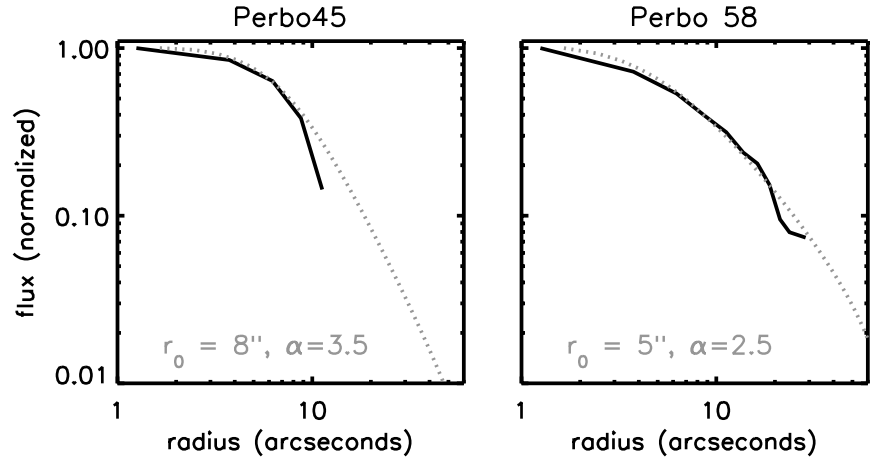

Figure 3. $3 \mathrm{~mm}$ flux profiles of Perbo45 (left) and Perbo58 (right), shown in solid black lines. The flux profiles of model cores with density profiles given by Equation (3) are shown in dotted gray lines. The inner point on the observed mass profiles is placed at 1 .' 25 , which is half of the HWHM of the typical beam size of the $3 \mathrm{~mm}$ observations. Values of $r_{0}$ and $\alpha$, as described in Section 3.1.2, are given in each panel.

we measure the flux profile in annuli centered on the positions of Perbo45 and Perbo58 given in Table 3. We then calculate the flux profile that would be observed for a spherical core with the density profile given by Equation (3) assuming that the cores are isothermal and have constant dust emissivities. We normalize the observed and model fluxes such that the flux at the center of the core is equal to unity, so the $n_{0}$ term in Equation (3) is not meaningful. We show in Figure 3 that under the stated assumptions of the dust properties and geometries that a density profile like that given in Equation (3) does predict flux profiles that are similar to those observed at $3 \mathrm{~mm}$ for values of $r_{0}$ in the range of $5^{\prime \prime}-8^{\prime \prime}$ and $\alpha$ in the range 2.5-3.5.

The model density profile that we use to fit the observed flux profiles of Perbo45 and Perbo58 does not provide a unique solution to what the true density profiles of these core are, and other models that have a flat inner profile that steepens at larger radii can be consistent with our observations. If one uses different assumptions about the temperature profile and allows the emissivity to vary with radius, it might even be possible to fit the $3 \mathrm{~mm}$ flux profile with a power-law density profile. What we have shown here is that the density profiles of Perbo 45 and Perbo58 are not necessarily different from typical starless cores, and the reason that they are detected in our survey while other cores are not is most likely due to their relatively large masses for their size.

\subsection{Detection Rate}

Of the 11 starless cores that were observed with CARMA, 2 were detected (Perbo45 and Perbo58) and 9 were not. Neither of the detected starless cores are seen to break into multiple components. The non-detection of the majority of the starless cores is not due to low sensitivity (the median upper limit for the non-detections is $0.2 M_{\odot}$-see Section 3.1.1 for details) but instead is likely the result of a lack of compact sub-structures within the cores. If all of the mass in each of the non-detected cores had been contained within a few compact structures, every core would have been detected in the CARMA observations (see Section 3.1.1 for an explanation of how the mass upper limits in Table 3 were derived). For example, the median $3 \sigma$ upper limit on the mass of the non-detected cores is a factor of 7 smaller than the core mass derived from the Bolocam map. Therefore, we argue that the non-detection of multiple components in all 11 cores in our sample is a significant result. Furthermore, given the high rate of multiplicity in Class 0 and Class I protostars
(Looney et al. 2000) and the low rate of multiplicity in starless cores, we suggest that fragmentation into binaries takes place during the collapse of a prestellar core or during the Class 0 stage.

The density profiles of many starless cores have been measured from (sub)millimeter continuum emission maps and it has generally been found that the density profiles are consistent with an $r^{-2}$ power law at large radii that flattens toward the center of the core (e.g., Ward-Thompson et al. 1994, 1999; Shirley et al. 2000). To study the density profiles of the starless cores in our sample, we use the mass and radius derived from the $1.1 \mathrm{~mm}$ observations to predict the $3 \mathrm{~mm}$ flux at the center of each core with the assumptions that the density profile goes as $r^{-2}$, that the emissivity spectral index is given by $\beta=2$, and that the cores are isothermal. We would have been able to detect (at the $3 \sigma$ level) all 11 cores in our sample if these assumptions were valid, but in fact we only detect two starless cores. As noted in Section 3.1.1, if $\beta=1$ then the detection rate of cores, and any substructure in them, would be easier than if $\beta=2$. Because we expect starless cores to be colder in their centers than at the edges, the flux profile of a "real" starless core will be somewhat flatter than our isothermal model would predict. Nevertheless, we conclude that the density profiles for the majority of the starless cores in our sample are flatter than an $r^{-2}$ profile at their centers, in agreement with other surveys.

The low rate of $3 \mathrm{~mm}$ continuum detections toward 11 of the brightest $\left(>200 \mathrm{mJy}\right.$ beam $^{-1}$ at $1.1 \mathrm{~mm}$ with Bolocam; Enoch et al. 2008) in this study is in agreement with the results of Olmi et al. (2005) who detect only one out of six dense cores in Perseus. The largest angular scale to which the interferometer was sensitive and the median noise in the maps in this study $\left(30^{\prime \prime}-40^{\prime \prime}\right.$ and $0.7 \mathrm{mJy}$ beam $\left.^{-1}\right)$ are similar to that in Olmi et al. $\left(2005,20^{\prime \prime}-40^{\prime \prime}\right.$ and $1-1.5 \mathrm{mJy}$ beam $\left.^{-1}\right)$. There is no overlap in the sample of sources mapped in this study and in Olmi et al. (2005).

There are 67 starless cores detected by Enoch et al. (2008) in the Perseus molecular cloud, of which we observed 11. Because we selected our sample based on their high peak fluxes, there is no reason to expect that the other starless cores in Perseus would have a higher detection rate at $3 \mathrm{~mm}$. The low rate $(0 / 11)$ of finding multiple $3 \mathrm{~mm}$ components at $5^{\prime \prime}$ resolution within $1.1 \mathrm{~mm}$ cores observed with $30^{\prime \prime}$ resolution is consistent with the $3 \mathrm{~mm}$ continuum OVRO observations of dense cores in Perseus carried out by Olmi et al. (2005), who found that none of the cores (out of six) were seen to be composed of multiple components. We argue that starless CMFs generated from single dish bolometer maps of Perseus (e.g., Enoch et al. 2008; Hatchell \& Fuller 2008) are not strongly biased by the blending of compact, close cores into more massive single cores.

\section{DISCUSSION}

Given that many stars are found in multiple systems (Duquennoy \& Mayor 1991) and that many protostars are also multiples (Looney et al. 2000; Haisch et al. 2004), it is worthwhile to examine when fragmentation takes place. If starless cores fragment before formation of an embedded object, then we would expect to be able to detect these sub-cores. On the other hand, if fragmentation takes place after the first protostar has formed, then a survey of starless cores will not find any evidence of fragmentation. In this study, we find that none of the 11 starless cores show any evidence of fragmentation. If these cores will eventually fragment, then this must happen during and/or after the collapse and formation of the first protostar. 
Late fragmentation on small scales, which is consistent with the results of this survey, is predicted by theories that create binaries from disk fragmentation (e.g., Bonnell \& Bate 1994; Kratter et al. 2010).

The observations described in this paper are in agreement with the results of other recent surveys in Perseus. Kirk et al. (2007) compared the line profiles of $\mathrm{C}^{18} \mathrm{O}$ (2-1) and $\mathrm{N}_{2} \mathrm{H}^{+}(1-0)$ toward a sample of 150 candidate dense cores, both prestellar and protostellar. They found that the $\mathrm{N}_{2} \mathrm{H}^{+}$lines, representing the densest gas within the core, display nearly thermal line widths, indicating little non-thermal activity at the core center. While the $\mathrm{C}^{18} \mathrm{O}$ gas shows significant nonthermal broadening, the velocity centroids of the two species are very similar, indicating that the connection between the cores and their environment is relatively calm. Interestingly, such quiescence across scales appears hard to reconcile with numerical simulations of turbulent clouds (Kirk et al. 2009) and may indicate a need for magnetic pressure support within the dense gas. Additionally, Jørgensen et al. (2007) compared the location of mid-infrared Spitzer sources and sub-millimeter cores in Perseus, finding excellent agreement between the deeply embedded protostars and their protostellar envelopes. Intriguingly, only 3 out of 40 cores were found to harbor multiple protostars, at the spatial resolution of Spitzer (MIPS $24 \mu \mathrm{m}$, $6^{\prime \prime}$ or $\left.\sim 1500 \mathrm{AU}\right)$. Thus, it appears that within Perseus there is little evidence for fragmentation within cores, at least until the protostellar stage, where the fragmentation length scale is smaller. This may be due to the smooth and quiescent manner in which the cores themselves appear to be forming.

We only detected significant $(\geqslant 5 \sigma)$ emission from 2 of the 11 starless cores in our sample and we put tight upper limits on the masses of multiple components in the other nine cores, from which we conclude that there is no substructure on $\sim 5^{\prime \prime}$ scales $(\sim 1200 \mathrm{AU})$ in the majority of starless cores. A smooth column density profile is predicted by many models of isolated star formation, such as the inside-out collapse model (Shu 1977) and ambipolar diffusion (Ciolek \& Mouschovias 1993). It is less clear if a smooth column density profile is consistent with cores created through turbulence, though some simulations show that turbulent molecular clouds do form quiescent cores with smooth column density profiles (Nakamura \& Li 2005; BallesterosParedes et al. 2003).

\section{SUMMARY}

We present a study of the $3 \mathrm{~mm}$ continuum emission from a sample of 11 of the brightest (at $1.1 \mathrm{~mm}$ ) starless cores in the Perseus molecular cloud. The data were primarily taken with CARMA at $\sim 5^{\prime \prime}$ resolution, with follow-up observations of one core taken with the SZA to improve the sensitivity to extended flux.

1. We detect two starless cores (Perbo45 and Perbo58), both of which are seen to contain a single, main component.

2. Based on the low detection rate $(0 / 11$ in this study and $0 / 6$ in Olmi et al. 2005) of multiple components within starless cores, we conclude that starless CMFs derived from bolometer maps of the Perseus molecular cloud (e.g., Enoch et al. 2008; Hatchell et al. 2007) are unlikely to be heavily biased by the blending of smaller, nearby cores into single sources at the coarser resolutions of single-dish maps.

3 . Based on the low detection rate of any components within dense cores (2/11 in this study and 1/6 in Olmi et al. 2005), we conclude that the majority of starless cores lack compact structures. More specifically, we find that density profiles in the inner portions of starless cores of the form $r^{-2}$ are inconsistent with our observations for the majority of the starless cores in our survey. This agrees with the results of previous surveys, which find that the density profiles of starless cores are flatter than $r^{-2}$ at small radii (e.g., Ward-Thompson et al. 1994, 1999; Shirley et al. 2000).

We thank John Carpenter, James Di Francesco, Brenda Matthews, and Sarah Sadavoy for helpful discussions. S.S. acknowledges support from a Plaskett Fellowship at the Herzberg Institute of Astrophysics. Support was provided to M.E. by NASA through the Spitzer Space Telescope Fellowship Program. D.J. is supported by a Natural Sciences and Engineering Research Council of Canada (NSERC) Discovery grant. Support for D.M. was provided by NASA through Hubble Fellowship grant HF-51259.01-A. We thank the CARMA staff, students, and postdocs for their help in making these observations. Support for CARMA construction was derived from the Gordon and Betty Moore Foundation, the Kenneth T. and Eileen L. Norris Foundation, the Associates of the California Institute of Technology, the states of California, Illinois, and Maryland, and the National Science Foundation. Ongoing CARMA development and operations are supported by the National Science Foundation under a cooperative agreement and by the CARMA partner universities. The JCMT is operated by the Joint Astronomy Centre on behalf of the Particle Physics and Astronomy Research Council of the United Kingdom, the Netherlands Organisation for Scientific Research, and the National Research Council of Canada. The CSO is supported by the NSF fund under contract AST 02-29008.

\section{REFERENCES}

Arce, H. G., \& Sargent, A. I. 2006, ApJ, 646, 1070

Ballesteros-Paredes, J., Klessen, R. S., \& Vázquez-Semadeni, E. 2003, ApJ, 592,188

Bonnell, I. A., \& Bate, M. R. 1994, MNRAS, 271, 999

Boss, A. P. 2002, ApJ, 568, 743

Boss, A. P. 2007, ApJ, 658, 1136

Ciolek, G. E., \& Mouschovias, T. C. 1993, ApJ, 418, 774

Crapsi, A., Caselli, P., Walmsley, M. C., \& Tafalla, M. 2007, A\&A, 470, 221

Di Francesco, J., Johnstone, D., Kirk, H., MacKenzie, T., \& Ledwosinska, E. 2008, ApJS, 175, 277

Duquennoy, A., \& Mayor, M. 1991, A\&A, 248, 485

Enoch, M. L., Evans, N. J., II, Sargent, A. I., Glenn, J., Rosolowsky, E., \& Myers, P. 2008, ApJ, 684, 1240

Enoch, M. L., et al. 2006, ApJ, 638, 293

Goodwin, S. P., \& Kroupa, P. 2005, A\&A, 439, 565

Goodwin, S. P., Nutter, D., Kroupa, P., Ward-Thompson, D., \& Whitworth, A. P. 2008, A\&A, 477, 823

Haisch, K. E., Jr., Greene, T. P., Barsony, M., \& Stahler, S. W. 2004, AJ, 127, 1747

Harvey, D. W. A., Wilner, D. J., Myers, P. C., \& Tafalla, M. 2003, ApJ, 597, 424

Hatchell, J., \& Fuller, G. A. 2008, A\&A, 482, 855

Hatchell, J., Fuller, G. A., Richer, J. S., Harries, T. J., \& Ladd, E. F. 2007, A\&A, 468, 1009

Hatchell, J., Richer, J. S., Fuller, G. A., Qualtrough, C. J., Ladd, E. F., \& Chandler, C. J. 2005, A\&A, 440, 151

Jørgensen, J. K., Johnstone, D., Kirk, H., \& Myers, P. C. 2007, ApJ, 656, 293

Keto, E., \& Caselli, P. 2010, MNRAS, 402, 1265

Kirk, H., Johnstone, D., \& Basu, S. 2009, ApJ, 699, 1433

Kirk, H., Johnstone, D., \& Di Francesco, J. 2006, ApJ, 646, 1009

Kirk, H., Johnstone, D., \& Tafalla, M. 2007, ApJ, 668, 1042

Kirk, J. M., Crutcher, R. M., \& Ward-Thompson, D. 2009, ApJ, 701, 1044

Kratter, K. M., Matzner, C. D., Krumholz, M. R., \& Klein, R. I. 2010, ApJ, 708, 1585

Lada, C. J. 2006, ApJ, 640, L63

Looney, L. W., Mundy, L. G., \& Welch, W. J. 2000, ApJ, 529, 477 
Matzner, C. D., \& McKee, C. F. 2000, ApJ, 545, 364

Motte, F., Andre, P., \& Neri, R. 1998, A\&A, 336, 150

Muchovej, S., et al. 2007, ApJ, 663, 708

Nakamura, F., \& Li, Z.-Y. 2005, ApJ, 631, 411

Nutter, D., \& Ward-Thompson, D. 2007, MNRAS, 374, 1413

Olmi, L., Testi, L., \& Sargent, A. I. 2005, A\&A, 431, 253

Ossenkopf, V., \& Henning, T. 1994, A\&A, 291, 943

Price, D. J., \& Bate, M. R. 2007, MNRAS, 377, 77

Sadavoy, S. I., et al. 2010, ApJ, 710, 1247

Sault, R. J., Teuben, P. J., \& Wright, M. C. H. 1995, Astron. Data Anal. Softw. Syst. IV, 77, 433

Schnee, S., Kauffmann, J., Goodman, A., \& Bertoldi, F. 2007, ApJ, 657, 838

Schnee, S., Rosolowsky, E., Foster, J., Enoch, M., \& Sargent, A. 2009, ApJ, 691,1754

Schnee, S., et al. 2010, ApJ, 708, 127
Shirley, Y. L., Evans, N. J., II, Rawlings, J. M. C., \& Gregersen, E. M. 2000, ApJS, 131, 249

Shirley, Y. L., Nordhaus, M. K., Grcevich, J. M., Evans, N. J., II, Rawlings, J. M. C., \& Tatematsu, K. 2005, ApJ, 632, 982

Shu, F. H. 1977, ApJ, 214, 488

Simpson, R. J., Nutter, D., \& Ward-Thompson, D. 2008, MNRAS, 391, 205

Steinacker, J., Pagani, L., Bacmann, A., \& Guieu, S. 2010, A\&A, 511, 9

Swift, J. J., \& Williams, J. P. 2008, ApJ, 679, 552

Tafalla, M., Myers, P. C., Caselli, P., \& Walmsley, C. M. 2004, A\&A, 416, 191

Ward-Thompson, D., Motte, F., \& Andre, P. 1999, MNRAS, 305, 143

Ward-Thompson, D., Scott, P. F., Hills, R. E., \& Andre, P. 1994, MNRAS, 268, 276

Williams, J. P., Lee, C. W., \& Myers, P. C. 2006, ApJ, 636, 952

Williams, J. P., \& Myers, P. C. 1999, ApJ, 518, L37

Williams, J. P., Myers, P. C., Wilner, D. J., \& di Francesco, J. 1999, ApJ, 513, L61 